\title{
Modular Integration of Hydrogel Neural Interfaces
}

\author{
Anthony Tabet, ${ }^{\bigcirc}$ Marc-Joseph Antonini, ${ }^{\bigcirc}$ Atharva Sahasrabudhe, Jimin Park, Dekel Rosenfeld, \\ Florian Koehler, Hyunwoo Yuk, Samuel Hanson, Jordan Stinson, Melissa Stok, Xuanhe Zhao, \\ Chun Wang, and Polina Anikeeva*
}

Cite This: ACS Cent. Sci. 2021, 7, 1516-1523

Read Online

ACCESS | W W Metrics \& More 回 Article Recommendations | st Supporting Information

ABSTRACT: Thermal drawing has been recently leveraged to yield multifunctional, fiber-based neural probes at near kilometer length scales. Despite its promise, the widespread adoption of this approach has been impeded by (1) material compatibility requirements and (2) labor-intensive interfacing of functional features to external hardware. Furthermore, in multifunctional fibers, significant volume is occupied by passive polymer cladding that so far has only served structural or electrical insulation purposes. In this article, we report a rapid, robust, and modular approach to creating multifunctional fiber-based neural interfaces using a solvent evaporation or entrapment-driven (SEED) integration process. This process brings together electrical, optical, and microfluidic modalities all encased

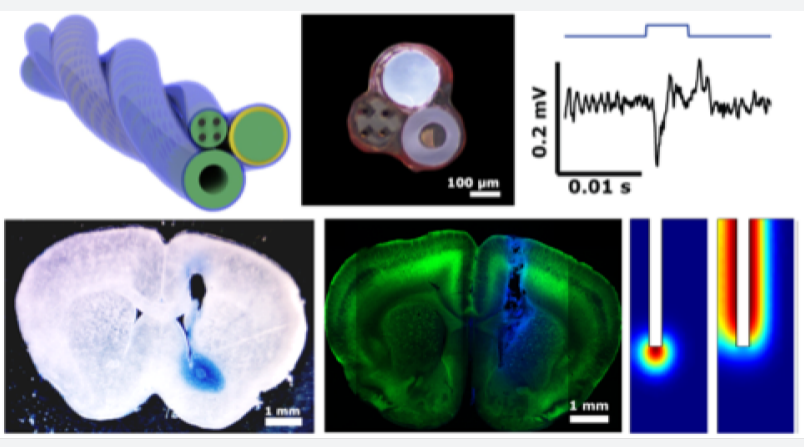
within a copolymer comprised of water-soluble poly(ethylene glycol) tethered to water-insoluble poly(urethane) (PU-PEG). We employ these devices for simultaneous optogenetics and electrophysiology and demonstrate that multifunctional neural probes can be used to deliver cellular cargo with high viability. Upon exposure to water, PU-PEG cladding spontaneously forms a hydrogel, which in addition to enabling integration of modalities, can harbor small molecules and nanomaterials that can be released into local tissue following implantation. We also synthesized a custom nanodroplet forming block polymer and demonstrated that embedding such materials within the hydrogel cladding of our probes enables delivery of hydrophobic small molecules in vitro and in vivo. Our approach widens the chemical toolbox and expands the capabilities of multifunctional neural interfaces.

\section{INTRODUCTION}

The fiber drawing process enables the fabrication of flexible neural probes that can simultaneously interrogate neuronal circuits via electrical, optical, and chemical modalities. During fiber drawing, a macroscopic model (the preform) of the desired probe is fabricated and drawn into hundreds of meters of fibers with microscale features. ${ }^{1,2}$ To date, these probes have enabled one-step optogenetics, ${ }^{3}$ in vivo photopharmacology, ${ }^{4}$ and in situ electrochemical synthesis of gaseous molecules for neuromodulation. ${ }^{5}$ Despite these advancements, this approach has several limitations. To be codrawable, the constituent materials need to have similar glass transition temperatures (for polymers) and melting temperatures (for metals). The resulting melt viscosities must also be compatible to obtain stable draw conditions necessary to maintain the crosssectional geometry of the preform. Additionally, while thermal drawing yields hundreds of meters of fiber at once, each individual centimeter-long device must be be manually connected to back-end hardware, a laborious process that currently represents the fabrication bottleneck. Furthermore, the polymer cladding of these fibers has only served passive structural or electrical insulation purposes, significantly adding to the device footprint with little added functionality.
Hydrogels are an attractive class of materials for neural interfaces. ${ }^{6}$ The mammalian brain itself is a weak hydrogel with a complex modulus $\mathrm{G}^{*}$ on the order of $1 \mathrm{kPa}^{7}$ While it has been shown that hydrogels alone can serve as neural interfaces, for example as optical waveguides ${ }^{8}$ or electrodes, ${ }^{9}$ their use in multifunctional neural probes has been more limited. ${ }^{10,11}$ Additionally, while hydrogels have been extensively used as depots for sustained release of bioactive molecules, ${ }^{12}$ this drug delivery capability has not yet been extended to multifunctional neural interfaces.

In this article, we complement thermal drawing with a solvent evaporation or entrapment-driven (SEED) integration process to create multifunctional hydrogel-based neural interfaces capable of eluting drugs and nanomaterials. Fibers comprising of optical waveguides, microfluidic channels, or electrode arrays were first drawn individually (Figure 1A-B).

Received: May 17, 2021

Published: August 28, 2021 
A

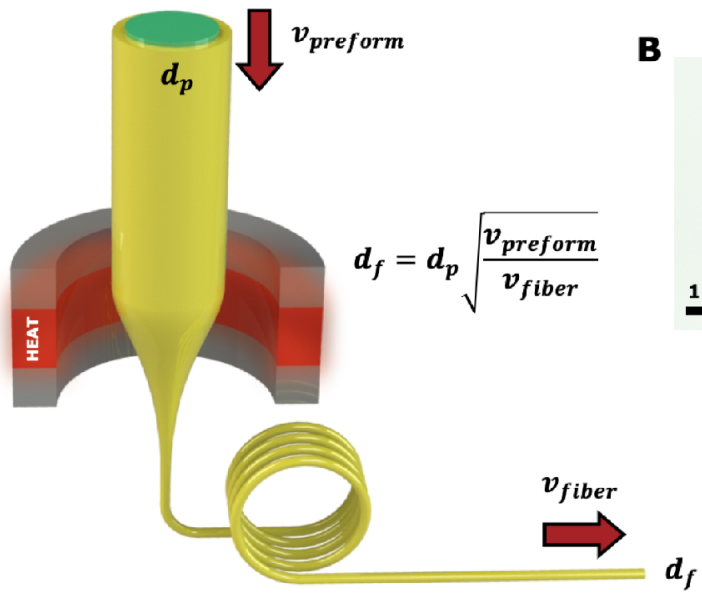

C

(i)

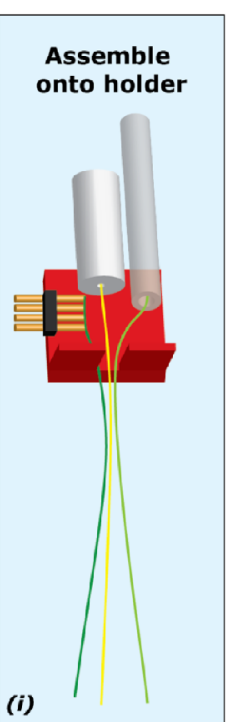

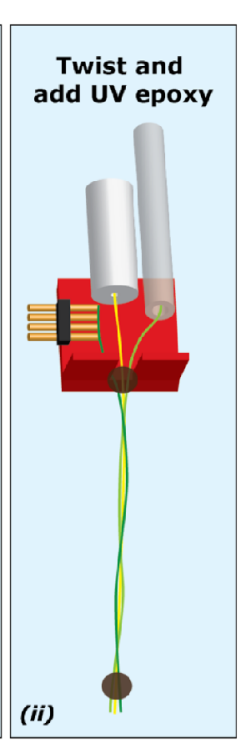

B

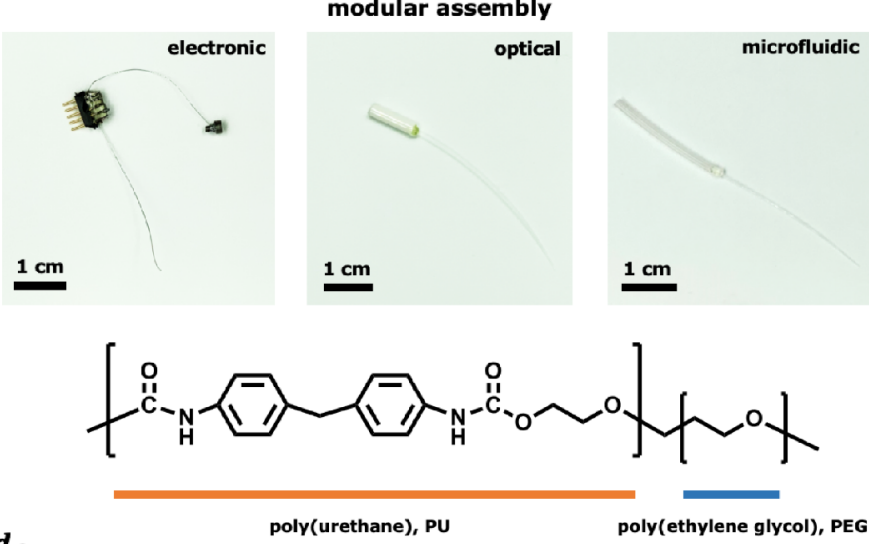

$\mathbf{E}$
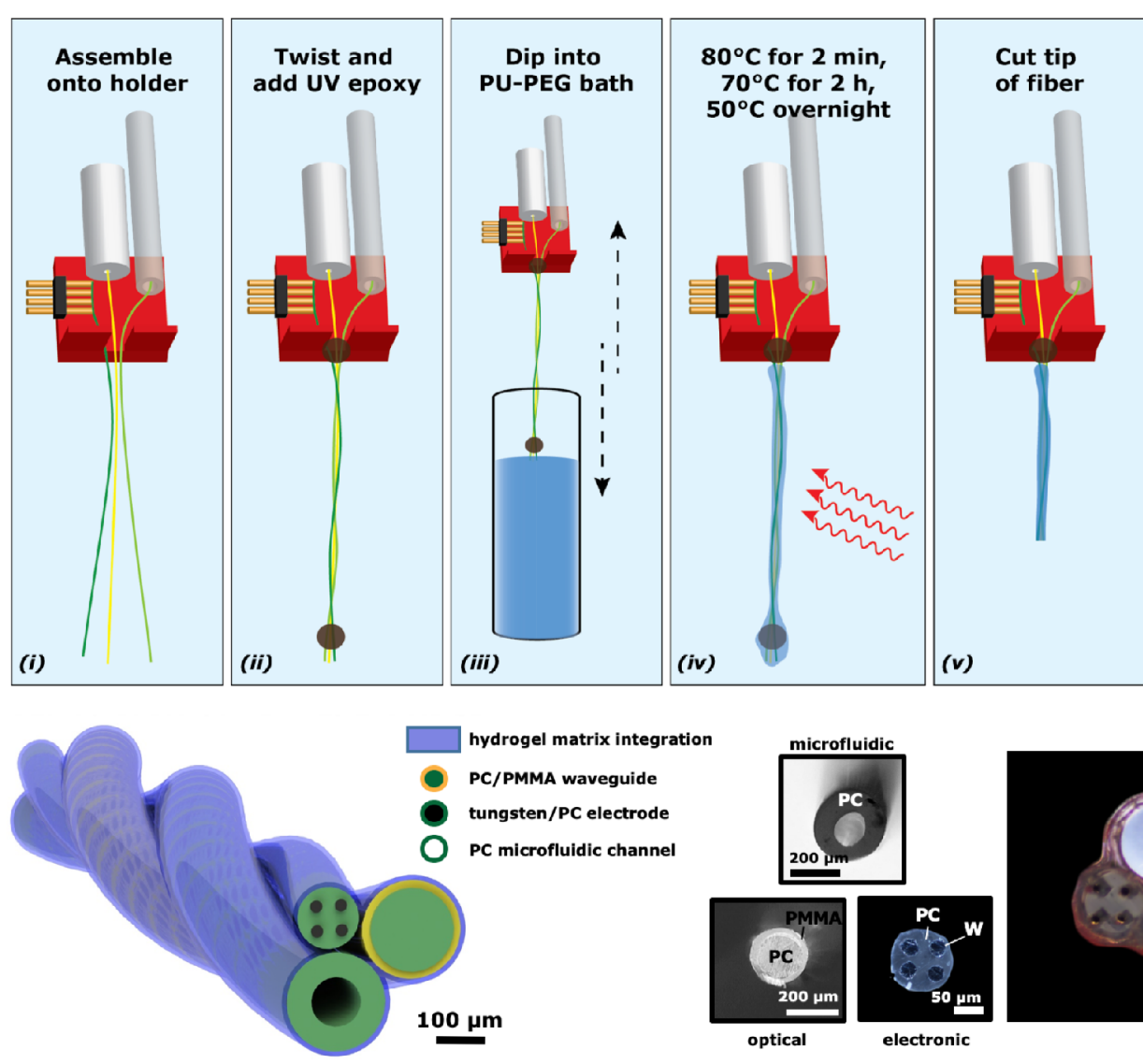

D
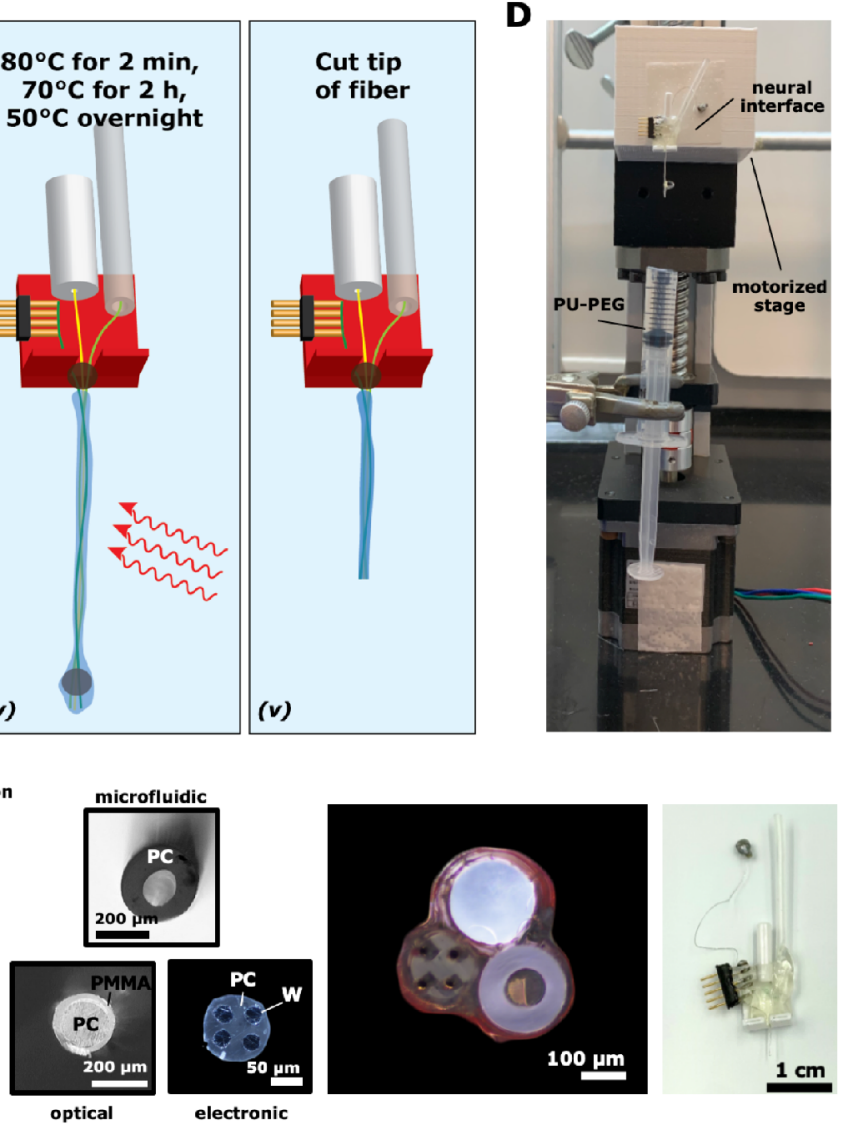

Figure 1. Creating multifunctional hydrogel neural interfaces with SEED integration. (A) Illustration of the thermal fiber drawing process of a preform into the electronic, optical, or microfluidic modules. (B) Three separate, fully connectorized modules (top) and chemical structure of the integrated poly(urethane)-poly(ethylene glycol) (PU-PEG) material (bottom). (C) Illustration of the SEED integration process steps: (i) the connectorized components are assembled, (ii) held in place with UV epoxy, (iii) then dip-coated into a PU-PEG bath dissolved in the ethanol/ water mixture. (iv) After heating, the fiber is (v) cut for in vivo studies. The case is colored red for contrast. (D) Image of the stepper motor stage used to control the dip-coating process. (E) Various components (left) of the assembled PU-PEG hydrogel and cross sections (right) of each component and the entire assembly.

The optical waveguide consisted of a poly(carbonate) (PC) core with a poly (methyl methacrylate) (PMMA) cladding $\left(n_{\mathrm{PC}}\right.$ $\left.=1.586, n_{\mathrm{PMMA}}=1.49\right)$, the recording electrode array fiber comprised four $25 \mu \mathrm{m}$ tungsten (W) wires within a PC cladding, and the microfluidic channel was a hollow PC fiber. By using a copolymer that forms a physical gel upon introduction of an aqueous medium, we combined these individual modalities into a single physical hydrogel-based neural interface (Figure 1C-D). This SEED integration process did not require any free radicals or other toxic byproducts ${ }^{11}$ and could accommodate a wide chemical toolbox previously inaccessible to multifunctional neural probes.

\section{RESULTS AND DISCUSSION}

To avoid sophisticated cleaning steps associated with potentially toxic radical initiators, we employed a copolymer of poly(ethylene glycol) tethered to water-insoluble poly(urethane) (PU-PEG), polymers with known biocompatibility 
A
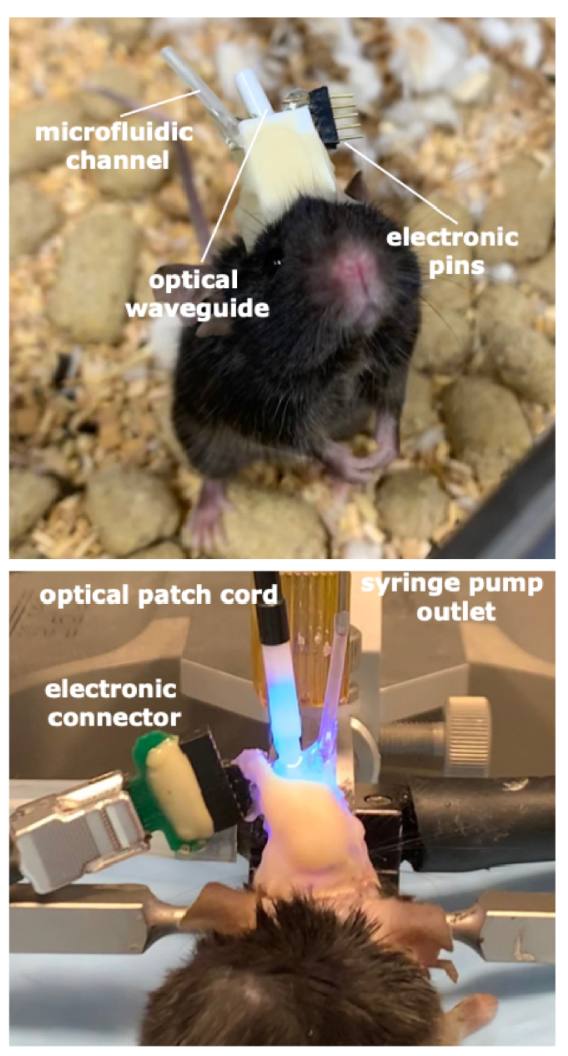

B
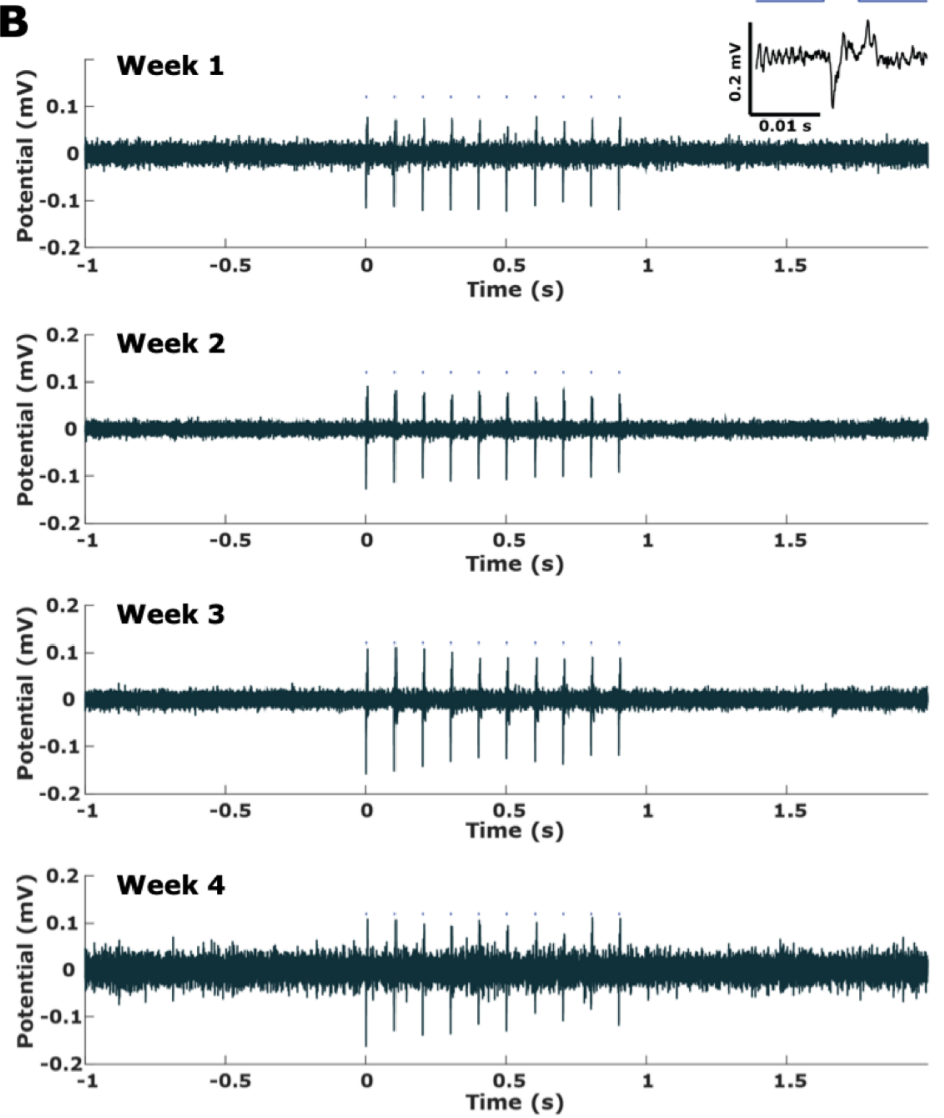

C

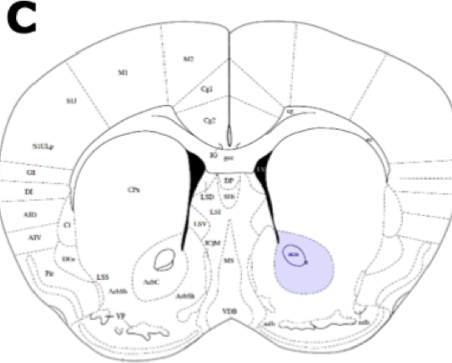

D
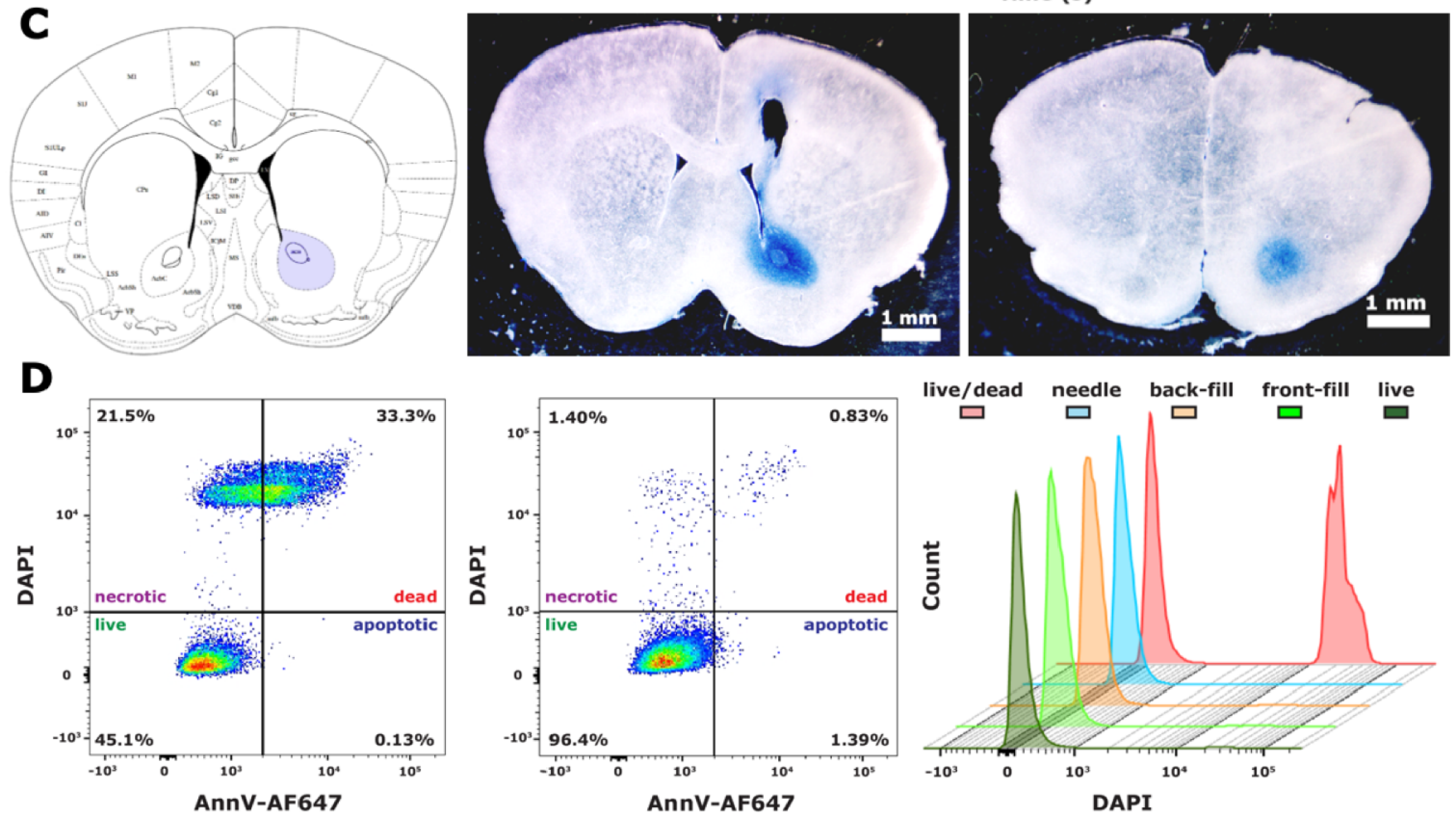

Figure 2. In vivo utilization of the hydrogel neural interface. (A) Image of wake transgenic Thy1-ChR2 mouse chronically implanted with the hydrogel neural interface (top). Image of optogenetically invoking action potentials in anesthetized transgenic Thy1-ChR2 mouse (bottom). (B) Chronic recording of optogenetically invoked action potentials in the nucleus accumbens (NAc) in a Thyl-ChR2 mouse. (C) Demonstrating release profile of drugs injected through the microfluidic channel using Evans blue dye. Brain atlas image with NAc highlighted in blue (left). Crosssection of a Thy1-ChR2 brain injected with $3 \mu \mathrm{L}$ of Evans blue dye at $33 \mathrm{~nL} / \mathrm{s}$ followed by fixation with $4 \%$ paraformaldehyde, showing clearly the location of the NAc bolus (middle). Cross-section of the same brain approximately $0.7 \mathrm{~mm}$ away, showing the periphery of the depot. (D) Flow cytometry data demonstrating the capability of these neural interfaces to deliver cells with high viability: a mixture of approximately $50 \%$ live and $50 \%$ dead or dying RAW-Blue murine macrophages (left) and cells injected through the microfluidic channel with the backfill method at $1 \mu \mathrm{L} / \mathrm{min}$ (middle). We obtained similar viability when comparing to injections with 26G NanoFil syringes and live cells left on ice (right). The histogram was normalized to the mode. 

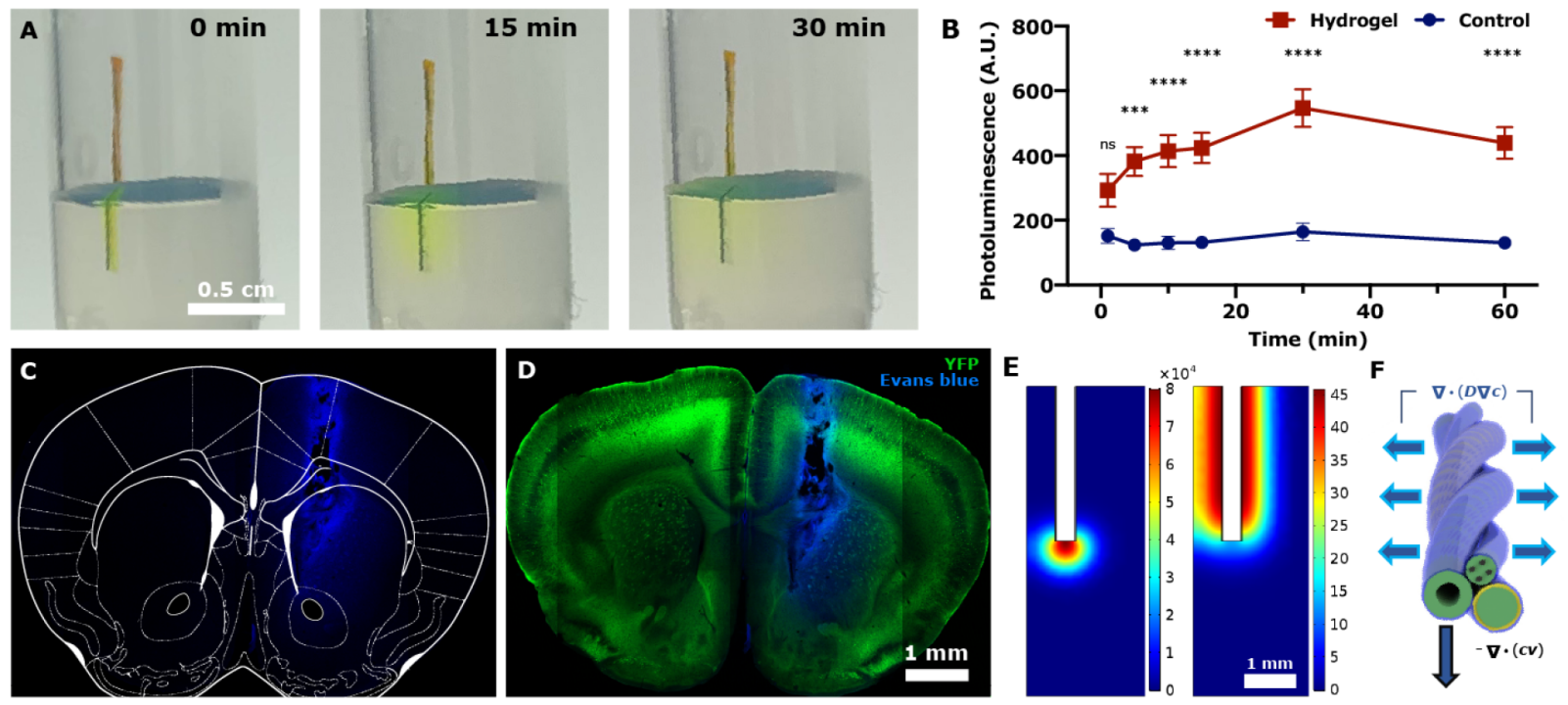

Figure 3. Drug delivery of molecules loaded into the hydrogel itself, independent of the microfluidic channel. (A) Images of the hydrogel neural interface loaded with fluorescein implanted into a $0.6 \%$ agarose phantom brain over time. (B) Transient release kinetics of fluorescein from integrated fibers with and without PU-PEG. The nonhydrogel control condition was the SEED integration with an equivalent concentration of fluorescein without hydrogel. In addition to depositing a lower concentration, the assembly did not stay integrated without the hydrogel in PBS. Statistical analysis was conducted using two-way ANOVA. $n=4$ in each group, ${ }^{* * *} P=0.0002, * * * * P<0.0001$. Error bars shown are \pm s.e.m. (CD) Evans blue dye was loaded into the hydrogel, and this device was implanted into the NAc of Thy1-ChR2 mice. After $72 \mathrm{~h}$, mice were perfused and sections were taken. We imaged these sections with confocal microscopy (4× objective, scans of regions stitched with FluoView software package). (E) Finite element modeling of mass transport of a small molecule from either the microfluidic channel (left) or hydrogel (right) 10 min after completion of the $3 \mu \mathrm{L}$ injection (same time point in both cases). (F) Illustration demonstrating both microfluidic and hydrogel-based drug delivery and the resulting convection vs diffusion driving forces, respectively.

routinely used in clinical implants and pharmaceuticals. ${ }^{13,14}$ Upon exposure to water, the PEG blocks facilitate hydration of the material while the hydrophobic forces between PU blocks prevent dissolution, resulting in a physical hydrogel. Since both blocks are soluble in ethanol, we first dissolved the copolymer in a 95\% ethanol solution to form a PU-PEG bath. We then brought the individual fiber components together in this bath and used a heat source to evaporate the solvent, resulting in an integrated assembly (Figure 1E). This integration creates hydrogel fibers that maintain structural integrity upon insertion in a phantom brain model, and after implantation in vivo (Figure S1). We then characterized the electrical, optical, and fluid delivery properties of these hydrogel-integrated probes (Figure S2-S4). The recording electrodes, ${ }^{2} 25 \mu \mathrm{m} \mathrm{W}$ wires, had impedance of $80 \mathrm{kOhm}$ at $1 \mathrm{kHz}$ which is well within the range suitable for extracellular recordings of neuronal potentials. ${ }^{1}$ We chose $\mathrm{W}$ over nickel chromium $(\mathrm{NiCr})$ used in tetrodes to avoid gold-plating, which is necessary to achieve sub-MOhm impedance, as that step would expose our hydrogel to an organic solvent. ${ }^{15}$ The $25 \mu \mathrm{m} \mathrm{W}$ electrodes were selected over the $12.5 \mu \mathrm{m}$ alternative because of $a>8$-times lower impedance (Figure S2). Optical losses in the PC/PMMA waveguide were measured as $0.76 \mathrm{~dB} / \mathrm{cm}$ loss at a $473 \mathrm{~nm}$ wavelength, which was consistent with previously observed losses in PC-core fibers and sufficient for optical neural excitation mediated by channelrhodopsin-2 (ChR2) (Figure S3). We observed an injection efficiency of $>90 \%$ for injection rates above $10 \mathrm{~nL} / \mathrm{s}$, confirming efficient fluid delivery through the microfluidic channels (Figure S4). Finally, using dynamic mechanical analysis (DMA), we assessed the bending stiffness of the hydrogel neural interfaces (Figure S5) and observed that these devices were flexible, in particular compared to other commonly used devices in neuroscience. ${ }^{3,16,17}$
We then evaluated the functionality of our hydrogel-based probes in transgenic mice broadly expressing ChR2 fused to a yellow fluorescent protein under the Thy1 promoter. ${ }^{18}$ ChR2 is a light-gated cation channel which, upon irradiation with blue light, causes neuronal depolarization and firing of action potentials. ${ }^{19}$ We chronically implanted our neural probes into the nucleaus accumbens (NAc) of Thy1-ChR2 mice (Figure $2 A)$. The NAc plays an important role in the cognitive processing of reward and motivation, and its aberrant function has been implicated in a wide range of mental disorders, including schizophrenia, substance addiction, and posttraumatic stress disorder. ${ }^{20}$ The polymer-based optical waveguide and $\mathrm{W}$ recording electrodes allowed us to optically stimulate and simultaneously record evoked (Figure 2B; (= $473 \mathrm{~nm}, 10 \mathrm{~Hz}, 5 \mathrm{~ms}$ pulse width, $20 \mathrm{~mW} / \mathrm{mm}^{2}$ ) and spontaneous activity (Figure S8) of NAc neurons in anesthetized mice. This activity was correlated with laser onset (jitter $=0.84 \mathrm{~ms}$, mean peak latency $=5.86 \mathrm{~ms}$, Figure S6). To confirm that the observed action potentials were not due to the photoelectrochemical (Becquerel) effect, ${ }^{21,22}$ we also performed electrophysiological recordings during optical stimulation in a chronically implanted Thy1-ChR2 mouse following euthanasia and observed no evoked activity (Figure S7). We then validated our microfluidic capabilities in vivo by delivering Evans blue dye ( $2 \%$ in sterile saline) into the NAc of Thy1-ChR2 mice. Ten minutes following injection, the animals were transcardially perfused with $4 \%$ paraformaldehyde, and widefield microscopy of brain slices revealed a dye depot formation in the NAc (Figure 2C-D).

Cell-based therapies remain an active area of research for fundamental understanding and future treatments of neurological diseases. ${ }^{23,24} \mathrm{We}$ assessed whether our devices are compatible with these emerging therapeutic approaches by 
evaluating the viability of cells following delivery through the integrated microfluidic channel. Depending on the application, the therapeutic cargo can be front-filled into the tip of the microfluidic channel and delivered during device implantation surgery $^{3}$ or can be backfilled days or weeks following chronic implantation $^{1}$ (Figure 2C). We evaluated the viability of RAWBlue macrophages (RBMs) using both delivery strategies via flow cytometric analysis with 4',6-diamidino-2-phenylindole (DAPI) and Annexin V (AnnV) conjugated to Alexa Fluor 647 (AnnV-AF647). As a DNA-binding dye, DAPI was used to probe the viability of cells as fluorescence is only observed when cell membrane integrity is lost during cell death. Annexin $\mathrm{V}$ was used to identify exposed aminophospholipid phosphatidylserine (PS). PS is normally maintained on the inner leaflet of the cell membrane under physiological conditions but becomes exposed during the early stages of regulated cell death and serves as a phagocytic signal. Together, these markers enable quantification of apoptotic and necrotic processes in response to cell stresses or treatments. We applied these markers to compare viability of cells delivered through the microfluidic channel within the hydrogel neural probe to those kept on ice, injected with a $26 \mathrm{G}$ NanoFil syringe, or killed via heat shock (Figure 2D). With either the front- or backfill approach, our devices retained RBM cell viability $>90 \%$ at a 1 $\mu \mathrm{L} / \mathrm{min}$ injection rate (Figures $2 \mathrm{E}$ and $\mathrm{S} 9$ ), suggesting that these probes can be used to deliver live cells directly into the central nervous system.

We next demonstrated that the hydrogel cladding enables delivery of small molecules along the entire length of the probe. We used fluorescein as a model drug and coloaded it into the hydrogel precursor PU-PEG ethanol solution. Since fluorescein is water-soluble, introduction into an agarose phantom brain results in diffusion of this molecule away from the hydrogel (Figures 3A and S10). Quantitative analysis of release into PBS shows a bolus release that peaks $30 \mathrm{~min}$ postinsertion (Figure 3B). We then demonstrated this capability in vivo by coloading Evans blue into the hydrogel and implanting the resulting neural probe into the NAc of Thy1-ChR2 mice (Figure 3C-D). Confocal microscopy images of brain slices revealed that hydrogel-loaded Evans blue has a different in vivo release profile compared to delivery through the microfluidic channel. Instead of convection-driven transport (first term on the right-hand side in eq 1) at the tip of the implant, Evans blue delivery is dominated by diffusiondriven transport (second term on the right-hand side in eq 1) (Figure $3 \mathrm{E}-\mathrm{F}$ ) and happens along the whole length of the implant. This additional drug delivery modality enabled by the hydrogel may be more advantageous for certain applications, such as the modulation of the foreign body response using antifibrotic drugs eluted along the length of implants. ${ }^{25}$

$$
\frac{\partial c}{\partial t}=-\nabla \cdot(c \mathbf{v})+\nabla \cdot(D \nabla c)+R
$$

Controlled drug delivery of hydrophobic small molecule drugs remains a formidable obstacle to their translational utility. Despite recent setbacks, ${ }^{26}$ emergent clinical applications of hydrophobic molecules, such as cannabinoids, have garnered renewed interest in their effective delivery. ${ }^{27}$ It was recently demonstrated that rationally designed polymers can overcome the delivery challenges of hydrophobic small molecules by, for example, forming nanodroplets that can carry these molecules into the cytosol. ${ }^{28}$ These custom polymers are melts at room temperature, with glass transition temperatures $>150{ }^{\circ} \mathrm{C}$, lower than that of PU-PEG, and are not codrawable with structural polymers typically used in fiber drawing of neural probes. SEED integration allows us to overcome these challenges and thus expands the drug delivery capabilities of neural interfaces. To enable delivery of hydrophobic compounds, a custom block copolymer (Scheme 1) of PEG and poly(caprolactone) (PCL)

Scheme 1. (top) Chemical Structure of the Customized Polyacetal PA11, a Block Copolymer, That Self-Emulsifies into Nanodroplets Capable of Drug Delivery of Hydrophobic Small Molecule Drugs, (bottom, left) Nanodroplet with the Center Shaded to Illustrate DrugLoading Capability in the Hydrophobic Region of the Polymer, and (bottom, right) Endosomal Escape of Hydrophobic Small Molecule Drugs
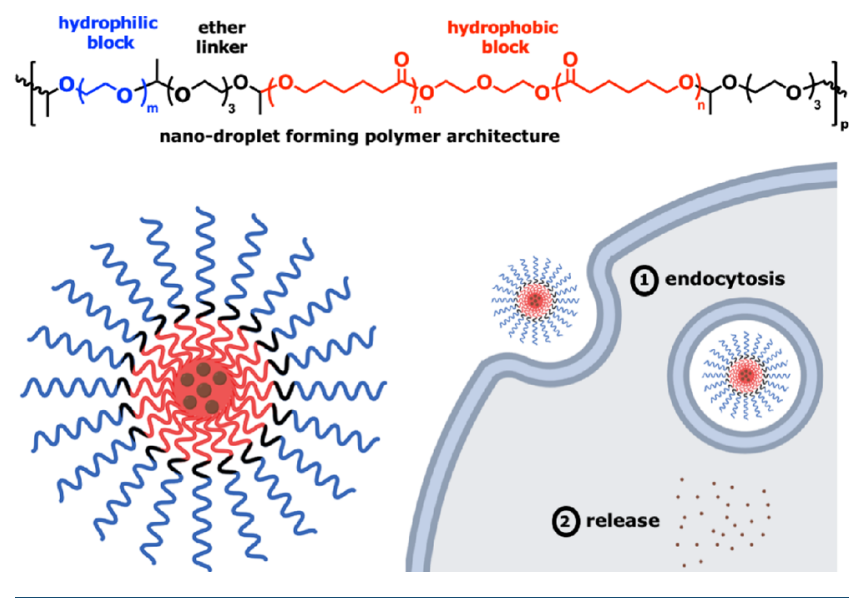

was synthesized with an acid-labile ether linkage. ${ }^{28}$ This poly(acetal), named PA11 (1:1 ratio of PEG:PCL), was blended with the PU-PEG precursor solution at a 3:17 ratio, and the resulting blend was applied during integration of a fiber assembly. We found that PA11 releases from the PU-PEG matrix and forms nanodroplets approximately $25 \mathrm{~nm}$ in diameter in saline solution under mild perturbations (Figure $4 \mathrm{~A}-\mathrm{C}$ ). As a positive control, we repeated the experiment with a thick film of the PU-PEG/PA11 blend and vigorously shook it overnight, which resulted in PA11 nanodroplets of similar size. Transmission electron microscopy (TEM) images of the dehydrated samples further corroborated formation and dimensions of these nanodroplets (Figure 4B). We then assessed the ability of PA11 to deliver hydrophobic compounds into aqueous media. We found that when the hydrophobic small molecule Nile Red was mixed in with the PA11:PU-PEG composite, PA11 could escape and carry the hydrophobic dye with it (Figure 4C). By immersing Nile Redloaded hydrogel fibers with and without PA11 into PBS and measuring the fluorescence after gentle shaking overnight, we observed release of the hydrophobic small molecule only when PA11 was coloaded into the hydrogel (Figure 4D-E). To further illustrate this functionality in the context of neurobiology, we incubated primary rat dorsal root ganglion (DRGs; sensory neuronal structures) with Nile Red in the presence or absence of PA11 (Figure 4F and S11). We found that, after a $24 \mathrm{~h}$ incubation with PA11, 96\% of neurons were Nile Redpositive (Figure 4F). No dye was found in neuronal cytoplasm or nuclei in the absence of PA11 (Figures 4G and S11), suggesting that this polymer is sufficient for effective intraneuronal delivery of hydrophobic small molecule drugs. 

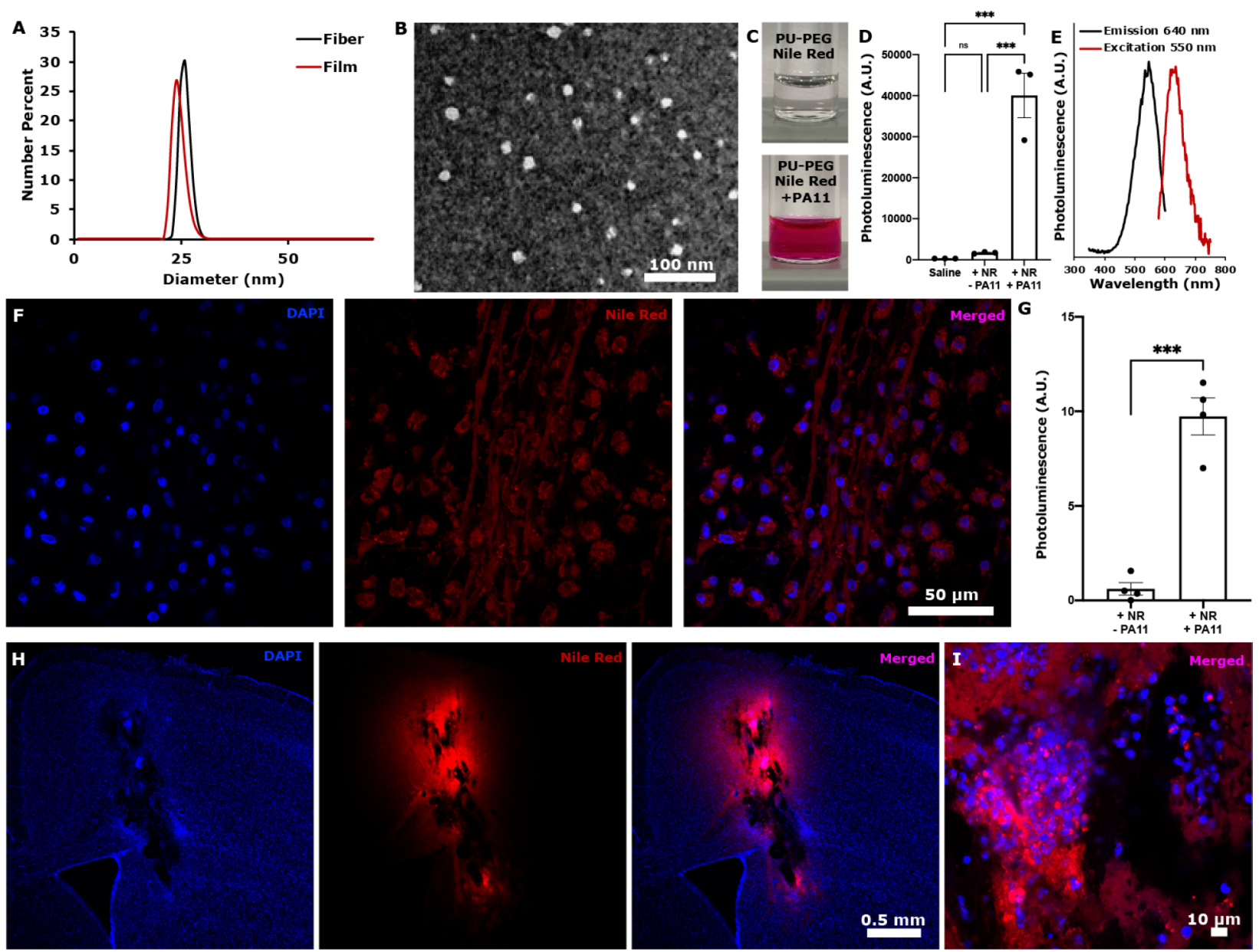

Figure 4. Drug delivery of nanoparticles and hydrophobic small molecules loaded into the hydrogel. (A) Dynamic light scattering (DLS) data of the solution after elution of PA11 from PA11/PU-PEG-based fibers. The vial containing the fiber was gently perturbed over $24 \mathrm{~h}$. As a positive control, we vigorously shook a thick PA11/PU-PEG film in PBS. (B) Transmission electron microscopy (TEM) images of PA11 nanodroplets. (C) Supernatant from a film of PU-PEG loaded with Nile Red without (top) or with (bottom) PA11. (D) Quantification via fluorescence spectroscopy of Nile Red loaded into hydrogel neural interfaces with and without PA11. Statistical analysis was conducted using ordinary one-way ANOVA. $n=$ 3 in each group, ${ }^{* * *} P<0.001$ ( $P=0.0003$ vs saline, $P=0.0004$ vs PA11). Error bars shown are \pm s.e.m. (E) Full fluorescence spectra of the fiber supernatant when both Nile Red and PA11 are incorporated. Excitation sweep was obtained with a fixed emission wavelength of $640 \mathrm{~nm}$ (black). Emission sweep was obtained with a fixed excitation wavelength of $550 \mathrm{~nm}$. Arbitrary unit axes were rescaled for clarity. (F) Confocal micrographs $(60 \times)$ of primary rat dorsal root ganglion neurons (DRGs) coincubated with media containing both PA11 and Nile Red for $24 \mathrm{~h}$. (G) Quantification of intensity on the Nile Red channel compared to no PA11 control. Statistical analysis was conducted using unpaired $t$ test. $n=4$ in each group, $* * * P=0.0001$. ( $\mathrm{H}-\mathrm{I})$ In vivo demonstration of NR/PA11 elution in the NAc of C57BL/6 mice after $72 \mathrm{~h}$ at $(\mathrm{H}) 4 \times$ and $(\mathrm{I}) 60 \times$ magnification.

Finally, we extended this concept in vivo and demonstrated that tissue along an implant contained Nile Red $72 \mathrm{~h}$ after an implantation of a PA11/Nile Red-loaded hydrogel probe (Figure 4H-I).

Our lab recently reported hydrogel-based neural probes utilizing a UV-cured poly(acrylamide)-alginate based hydrogel. ${ }^{11}$ Although these devices enabled long-term electrophysiological and optogenetic studies, they had some limitations. Namely, assembly of these devices was labor intensive, required device fabrication in an oxygen-free environment due to oxygen-sensitive chemistry, and utilized neurotoxic radicals that had to be thoroughly eliminated through an extensive washing process prior to deployment in neuroscience applications. By contrast, SEED integration utilizing PU-PEG hydrogels is a robust and translatable method that overcomes all of these limitations and requires only a few minutes of active fabrication time. Additionally, unlike previous neural probes reported from our group that utilized passive cladding, ${ }^{1,3,5,11,17}$ devices fabricated with SEED integration feature active cladding which can be coloaded with both small molecule drugs and drug nanocarriers for delivery in vivo. These properties render these modular hydrogel neural interfaces well posed for fundamental and translational biological research.

\section{CONCLUSIONS}

By leveraging a SEED integration approach that employs amphiphilic copolymers, we created modular hydrogel neural interfaces capable of optogenetics, electrophysiology, and microfluidic delivery. We demonstrated that these devices are capable of delivering a variety of cargo, including cellular therapies with a high viability at fast injection rates. By loading model drugs or nanomaterials into the hydrogel itself, we also demonstrated a separate drug delivery modality with a unique driving force and release profile. We further showed the potential to extend this concept to new directions, including 
drug delivery of hydrophobic cargo. This platform expands the chemical toolbox and functionality available to soft neural interfaces.

\section{ASSOCIATED CONTENT}

\section{SI Supporting Information}

The Supporting Information is available free of charge at https://pubs.acs.org/doi/10.1021/acscentsci.1c00592.

Experimental details and supporting Figures S1-S11 (PDF)

\section{AUTHOR INFORMATION}

\section{Corresponding Author}

Polina Anikeeva - Department of Materials Science and Engineering, McGovern Institute for Brain Research, and Department of Brain and Cognitive Sciences, Massachusetts Institute of Technology, Cambridge, Massachusetts 02139, United States; Research Laboratory of Electronics, Massachusetts Institute of Technology, Cambridge,, Massachusetts 02139, United States; 이이이.org/00000001-6495-5197; Email: anikeeva@mit.edu

\section{Authors}

Anthony Tabet - Department of Chemical Engineering, McGovern Institute for Brain Research, and Koch Institute For Integrative Cancer Research, Massachusetts Institute of Technology, Cambridge, Massachusetts 02139, United States; Research Laboratory of Electronics, Massachusetts Institute of Technology, Cambridge,, Massachusetts 02139, United States

Marc-Joseph Antonini - Harvard/MIT Health Science \& Technology Graduate Program, Cambridge, Massachusetts 02139, United States; Research Laboratory of Electronics, Massachusetts Institute of Technology, Cambridge, Massachusetts 02139, United States; McGovern Institute for Brain Research, Massachusetts Institute of Technology, Cambridge, Massachusetts 02139, United States

Atharva Sahasrabudhe - Department of Chemistry and McGovern Institute for Brain Research, Massachusetts Institute of Technology, Cambridge, Massachusetts 02139, United States; Research Laboratory of Electronics, Massachusetts Institute of Technology, Cambridge, Massachusetts 02139, United States

Jimin Park - Department of Materials Science and Engineering and McGovern Institute for Brain Research, Massachusetts Institute of Technology, Cambridge, Massachusetts 02139, United States; Research Laboratory of Electronics, Massachusetts Institute of Technology, Cambridge, Massachusetts 02139, United States

Dekel Rosenfeld - McGovern Institute for Brain Research, Massachusetts Institute of Technology, Cambridge, Massachusetts 02139, United States; Research Laboratory of Electronics, Massachusetts Institute of Technology, Cambridge,, Massachusetts 02139, United States

Florian Koehler - McGovern Institute for Brain Research, Massachusetts Institute of Technology, Cambridge, Massachusetts 02139, United States; Research Laboratory of Electronics, Massachusetts Institute of Technology, Cambridge,, Massachusetts 02139, United States

Hyunwoo Yuk - Department of Mechanical Engineering, Massachusetts Institute of Technology, Cambridge, Massachusetts 02139, United States; (1) orcid.org/00000003-1710-9750
Samuel Hanson - Department of Biomedical Engineering, University of Minnesota, Minneapolis, Minnesota 55455, United States

Jordan Stinson - Department of Biological Engineering and Koch Institute For Integrative Cancer Research, Massachusetts Institute of Technology, Cambridge, Massachusetts 02139, United States

Melissa Stok - Department of Materials Science and Engineering, Massachusetts Institute of Technology, Cambridge, Massachusetts 02139, United States

Xuanhe Zhao - Department of Mechanical Engineering, Massachusetts Institute of Technology, Cambridge, Massachusetts 02139, United States; orcid.org/00000001-5387-6186

Chun Wang - Department of Biomedical Engineering, University of Minnesota, Minneapolis, Minnesota 55455, United States; $\odot$ orcid.org/0000-0003-2634-4795

Complete contact information is available at:

https://pubs.acs.org/10.1021/acscentsci.1c00592

\section{Author Contributions}

OA.T. and M.-J.A. contributed equally.

\section{Notes}

All animal experiments in this work were approved by the MIT Committee on Animal Care.

The authors declare no competing financial interest.

\section{ACKNOWLEDGMENTS}

A.T. thanks the National Science Foundation Graduate Research Fellowship and the Paul and Daisy Soros Fellowship for funding support. A.T. thanks Prof. K. Dane Wittrup for continuous mentorship throughout this project. A.T. also thanks thesis committee members Prof. Isaac Chiu, Prof. Kwanghun Chung, and Prof. Sean Lawler for thoughtful discussions and valuable feedback. The authors additionally thank Dr. Ameya Kirtane for support with DLS measurements, Dr. Andres Canales for assistance in constructing the integration tower, Georgios Varnavides for assistance with image processing, and Prof. James Frank for fruitful conversations on hydrophobic small molecules. This work was supported in part by the National Institute of Neurological Disorders and Stroke (R01-NS115025-01A1, P.A.), National Science Foundation (NSF) Center for Materials Science and Engineering (DMR-1419807, P.A.), NSF Center for Neurotechnology (EEC-1028725, P.A.), and the McGovern Institute for Brain Research at MIT (P.A.).

\section{REFERENCES}

(1) Canales, A.; et al. Multifunctional fibers for simultaneous optical, electrical and chemical interrogation of neural circuits in vivo. Nat. Biotechnol. 2015, 33, 277-284.

(2) Antonini, M.-J. Customizing MRI-Compatible Multifunctional Neural Interfaces through Fiber Drawing. Adv. Funct. Mater. 2021, 2104857.

(3) Park, S.; et al. One-step optogenetics with multifunctional flexible polymer fibers. Nat. Neurosci. 2017, 20, 612-619.

(4) Frank, J. A.; Antonini, M.-J.; Chiang, P.-H.; Canales, A.; Konrad, D. B.; Garwood, I. C.; Rajic, G.; Koehler, F.; Fink, Y.; Anikeeva, P. Vivo Photopharmacology Enabled by Multifunctional Fibers. ACS Chem. Neurosci. 2020, 11, 3802.

(5) Park, J.; et al. In situ electrochemical generation of nitric oxide for neuronal modulation. Nat. Nanotechnol. 2020, 15, 690-697. 
(6) Spencer, K. C.; Sy, J. C.; Ramadi, K. B.; Graybiel, A. M.; Langer, R.; Cima, M. J. Characterization of Mechanically Matched Hydrogel Coatings to Improve the Biocompatibility of Neural Implants. Sci. Rep. 2017, DOI: 10.1038/s41598-017-02107-2.

(7) Tabet, A.; Mommer, S.; Vigil, J. A.; Hallou, C.; Bulstrode, H.; Scherman, O. A. Mechanical Characterization of Human Brain Tissue and Soft Dynamic Gels Exhibiting Electromechanical Neuro-Mimicry. Adv. Healthcare Mater. 2019, 8, 1900068.

(8) Choi, M.; Choi, J. W.; Kim, S.; Nizamoglu, S.; Hahn, S. K.; Yun, S. H. Light-guiding hydrogels for cell-based sensing and optogenetic synthesis in vivo. Nat. Photonics 2013, 7, 987.

(9) Nam, J. Supramolecular Peptide Hydrogel-Based Soft Neural Interface Augments Brain Signals through a Three-Dimensional Electrical Network. ACS Nano 2020, 14, 664.

(10) Sung, C.; Jeon, W.; Nam, K. S.; Kim, Y.; Butt, H.; Park, S. Multimaterial and multifunctional neural interfaces: From surface-type and implantable electrodes to fiber-based devices, 2020.

(11) Park, S.; Yuk, H.; Zhao, R.; Yim, Y. S.; Woldeghebriel, E. W.; Kang, J.; Canales, A.; Fink, Y.; Choi, G. B.; Zhao, X.; Anikeeva, P. Adaptive, Multifunctional Hydrogel Hybrid Probes for Long-Term Sensing and Modulation of Neural Activity. Nat. Commun. 2021, DOI: $10.1038 / \mathrm{s} 41467-021-23802-9$.

(12) Li, J.; Mooney, D. J. Designing hydrogels for controlled drug delivery. Nat. Rev. Mater. 2016, DOI: 10.1038/natrevmats.2016.71.

(13) Baden, L. R. Efficacy and Safety of the mRNA-1273 SARSCoV-2 Vaccine. N. Engl. J. Med. 2021, 384, 403.

(14) Wang, W.; Wang, C. The Design and Manufacture of Medical Devices; 2012.

(15) Ferguson, J. E.; Boldt, C.; Redish, A. D. Creating lowimpedance tetrodes by electroplating with additives. Sens. Actuators, A 2009, 156, 388.

(16) Chen, R.; Canales, A.; Anikeeva, P. Neural recording and modulation technologies; 2017.

(17) Frank, J. A.; Antonini, M. J.; Anikeeva, P. Next-generation interfaces for studying neural function; 2019.

(18) Arenkiel, B. R.; Peca, J.; Davison, I. G.; Feliciano, C.; Deisseroth, K.; Augustine, G. J. J.; Ehlers, M. D.; Feng, G. In Vivo Light-Induced Activation of Neural Circuitry in Transgenic Mice Expressing Channelrhodopsin-2. Neuron 2007, 54, 205.

(19) Boyden, E. S.; Zhang, F.; Bamberg, E.; Nagel, G.; Deisseroth, K. Millisecond-timescale, genetically targeted optical control of neural activity. Nat. Neurosci. 2005, 8, 1263.

(20) Mavridis, I. The role of the nucleus accumbens in psychiatric disorders; 2015.

(21) Cardin, J. A.; Carlén, M.; Meletis, K.; Knoblich, U.; Zhang, F.; Deisseroth, K.; Tsai, L. H.; Moore, C. I. Targeted optogenetic stimulation and recording of neurons in vivo using cell-type-specific expression of Channelrhodopsin-2. Nat. Protoc. 2010, 5, 247.

(22) Kozai, T. D.; Vazquez, A. L. Photoelectric artefact from optogenetics and imaging on microelectrodes and bioelectronics: new challenges and opportunities. J. Mater. Chem. B 2015, 3, 4965.

(23) Teng, Y. D.; Lavik, E. B.; Qu, X.; Park, K. I.; Ourednik, J.; Zurakowski, D.; Langer, R.; Snyder, E. Y. Functional recovery following traumatic spinal cord injury mediated by a unique polymer scaffold seeded with neural stem cells. Proc. Natl. Acad. Sci. U. S. A 2002, 99, 3024.

(24) Parmar, M.; Grealish, S.; Henchcliffe, C. The future of stem cell therapies for Parkinson disease. Nat. Rev. Neurosci. 2020, 21, 103.

(25) Farah, S. Long-term implant fibrosis prevention in rodents and non-human primates using crystallized drug formulations. Nat. Mater. 2019, 18, 892.

(26) Lin, A. Off-target toxicity is a common mechanism of action of cancer drugs undergoing clinical trials. Sci. Transl. Med. 2019, 11, eaaw0064.

(27) Sarott, R. C.; Viray, A. E.; Pfaff, P.; Sadybekov, A.; Rajic, G.; Katritch, V.; Carreira, E. M.; Frank, J. A. Optical Control of Cannabinoid Receptor 2-Mediated Ca2+Release Enabled by Synthesis of Photoswitchable Probes. J. Am. Chem. Soc. 2021, 143, 736.
(28) Zhang, L.; Zhang, Z.; Wang, W.; Tabet, A.; Hanson, S.; Zhang, L.; Zhu, D.; Wang, C. Polymer-Based Dual-Responsive SelfEmulsifying Nanodroplets as Potential Carriers for Poorly Soluble Drugs. ACS Applied Bio Materials 2021, 4, 4441. 